\title{
Development of Disaster-Responding Special-Purpose Machinery: Results of Experiments
}

\author{
Jin Tak Kim ${ }^{1}$, Sangshin Park ${ }^{1}$, SangChul Han ${ }^{1}$, Jinhyeon Kim ${ }^{1}$, Hyogon Kim² ${ }^{2}$ Young-Ho \\ Choi $^{2}$, Jaehong Seo ${ }^{1,3}$, Sanguk Chon ${ }^{1,4}$, Jungyeong Kim ${ }^{1,4}$, and Jungsan Cho ${ }^{1}$ \\ ${ }^{1}$ Applied Robot R\&D Department, Research Institute of Convergence Technology, Korea \\ Institute of Industrial Technology \\ ${ }^{2}$ Intelligent Robotics R\&D Division, Korea Institute of Robotics and Technology \\ Convergence \\ ${ }^{3}$ Intelligent Robot Engineering, University of Science \& Technology \\ ${ }^{4}$ Robotics and Virtual Engineering, University of Science \& Technology
}

May 3, 2021

\begin{abstract}
The frequent occurrence of disasters has prompted the development of efficient disaster-responding equipment. This study deals with the design of special-purpose machinery and its performance assessment. We defined scenarios through the environmental analysis of disaster situations and expert consulting. We also formulated a set of design specifications by analyzing the objectives of the tasks that must be performed at disaster sites, based on simulated scenarios and existing disaster response machinery. The disaster-responding special-purpose machinery was designed to perform various tasks and display rapid movement in disaster situations. And this paper presents the control structure configured to operate the developed machinery. The performance of the special-purpose machinery was assessed through different scenario tests.
\end{abstract}

\section{INTRODUCTION}

Large disasters have frequently been occurring across the world due to urbanization and climate change. Rapid response within the golden hour is essential to minimize the loss of lives and secondary damage at the disaster sites. Firefighters generally enter the site and perform rescue operations because secondary damage factors exist in these areas due to additional collapse and leakages. Large debris is dismantled or moved to secure the access roads and support the rescue operations of the firefighters. Although excavators are commonly used in such operations, it is difficult for them to perform different tasks in irregular environments after a disaster because they are optimized to dig the ground under ordinary circumstances. In addition, there are extensive differences in the workability of the excavators due to the varying skill level of the drivers. As a result, it takes a long time for the excavators to enter the site because they move by using the front and tend to swing due to their limited mobility. The anxiety of failing in situations that may compromise the safety of the driver also limits rapid responses. Thus, there is a need for equipment capable of agile movement and performing rapid precision work in irregular environments.

Various types of disaster response equipment are under development globally. The Korea Institute of Robotics and Technology (KIRO) developed an armored robot system that can be deployed in complex disaster sites (Jeong, Kim, Kim, Suh, \& Jin, 2017)(Park et al., 2018). KIRO developed a car frame for the dual-arm 
manipulator to remove heavy obstacles, cut walls made of sandwich panels, and protect passengers from falling objects, toxic gas, and high temperature.

Hitachi's ASTACO (Ishii, 2006)(Double Arm Working Machine - Hitachi Construction Machinery, n.d.) is an example of the symmetrical implementation of two working machines on the body of an existing excavator and is called "an excavator with two fronts." The large model (NEO) is primarily used for dismantling buildings. The equipment deployed in the Tokyo Fire Department weighs 7 tons and has symmetrical fronts - each of which comprises six joints.

Tmsuk's T-52 comprises two arms, each of which consists of 6 DOF. Each arm has an attachment that can perform different functions and also provides an additional DOF. The operator can directly ride and manipulate the equipment; however, it also supports remote control to perform rescue operations in locations that cannot be accessed by rescuers (Nishida et al., 2006).

The Guardian GT, manufactured by Sarcos (Salt Lake City, Utah, USA), is a dual-arm robot designed to efficiently transport heavy objects in an unstructured environment in industrial sites by modifying the system manufactured by Ditch Witch and attaching big arms (Sarcos Guardianß GT Robot, n.d.). The Guardian GT can be easily manipulated by beginners because it has a human-like structure and follows human movements.

KRAFT used a Brokk machine to design a manipulator with 6 DOF mounted on a mobile platform with two crawlers. It was designed for remote control operations at building demolition sites. Four outriggers can be unfolded in the front, rear, left, and right directions to prevent the manipulator from overturning during work (Montazeri \& Ekotuyo, 2016). Tadano's ROBOTOPS had a body with two arms and could walk on four legs and move using tracks (Tadano Robotops 2009, n.d.).

As shown in the examples mentioned above, various disaster-responding systems have been developed across the globe. It remains a challenge to combine manipulation skills with the ability to perform on rough terrain. Dual-arm manipulators that can move rapidly and simultaneously overcome the limitations of conventional excavators are being designed. 


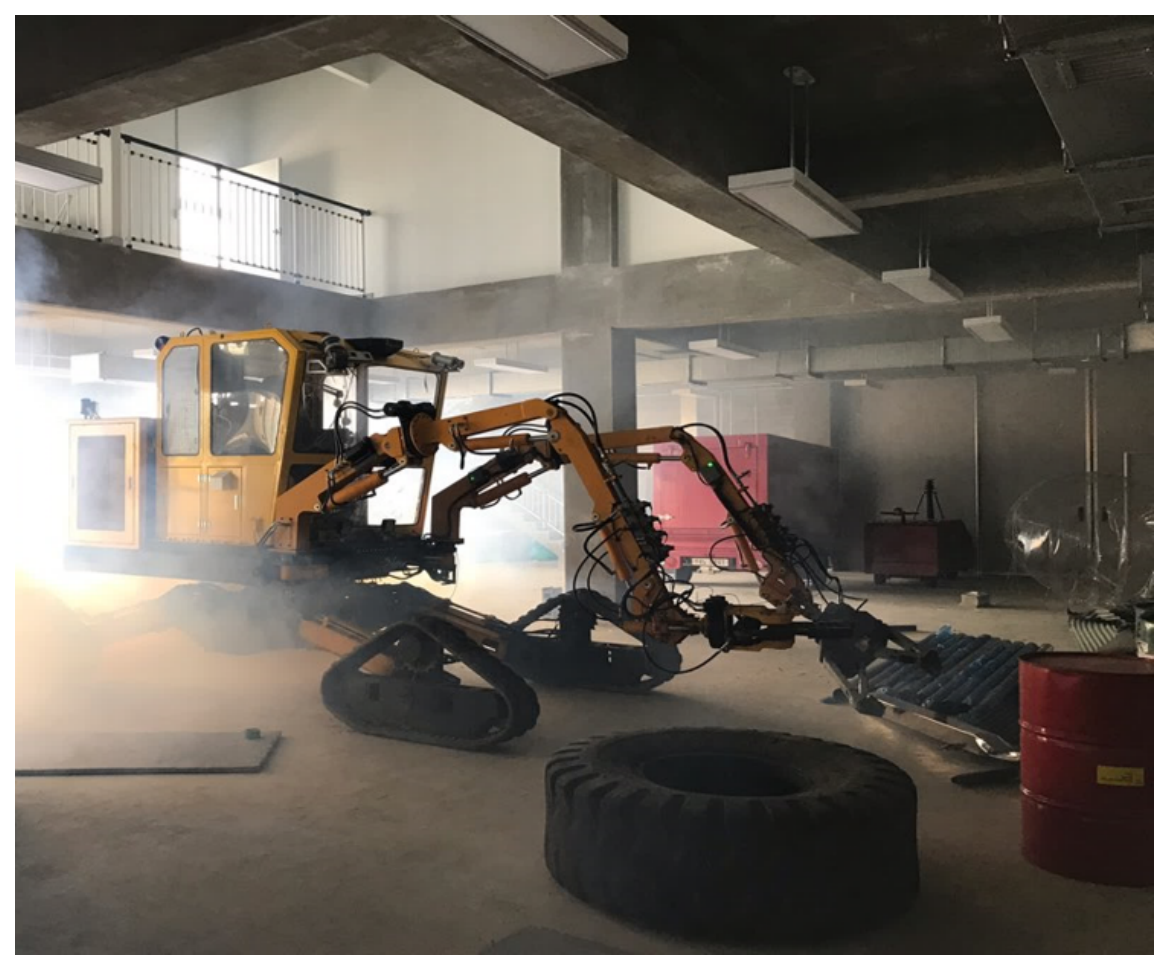

Figure 1: The disaster-responding special-purpose machinery

We analyzed the environment for potential disasters and developed certain scenarios with the consultation of experts. Following that, the objectives of the tasks to be performed and the capabilities of existing disaster-responding systems are analyzed, and a set of design specifications are derived. Special-purpose disaster-responding machinery, based on the derived design specifications, was developed while ensuring that the system can perform various tasks efficiently and move rapidly at disaster sites, as shown in Fig.1. The machinery developed in this study comprises symmetrical manipulators with 7 DOF on the left and right, with a multifunctional, multi-branch attachment on each manipulator. The driving system has four legs with $2 \mathrm{DOF}$ and an independently driven crawler at the end of each leg. The mainframe has a power pack and a swing joint. It consists of a single-person cabin and master devices to drive the system.

It is important for the system and the control methods to be designed according to the movement and workability of the drivers. This allows the system to perform complex tasks with fast movement at a disaster site with crowded debris. Unlike conventional excavators that control one 4 DOF manipulator by using two human arms, the disaster-responding special-purpose machinery proposed in the current study uses robotic technology to enable firefighters or other drivers to manipulate the system easily and intuitively. Thus, this study proposes the development of a special-purpose machinery system and analyzes its performance in a simulated disaster environment.

This paper is organized as follows. Chapter 2 describes the scenarios derived through an analysis of disaster environments and the system specifications required to tackle these scenarios. The dual-arm manipulators, driving system, cabin, hydraulic system, and control network system are discussed in Chapter 3. Chapter 4 includes the control architecture designed to control the system. Chapter 5 describes the details of the experiments conducted in this study, and the concluding remarks are presented in Chapter 6 . 
Table 1: Objectives of scenario tasks

\begin{tabular}{lll}
\hline Type & Task & Goal \\
\hline Driving & Flat surface & $4.1 \mathrm{~km} / \mathrm{h}$ \\
& Slope & Lateral side slope $15^{\circ}$ \\
& Longitudinal slope $11^{\circ}$ \\
& Uneven terrain & Driving on an irregular road surface with $10 \mathrm{~cm}$ or smaller debris \\
& Overcoming steps & Passing through a $50 \mathrm{~cm}$ high obstacle \\
\hline Work & Lifting & 400 kg at maximum reach \\
& Crushing & Crushing a concrete block \\
& Grabbing (Lifting) & Lifting a tire weighing $200 \mathrm{~kg}$ \\
& Pushing & Pushing drums \\
& Cutting & Cutting a $20 \mathrm{~mm}$ thick steel bar \\
& Spreading & Tearing open a $0.5 \mathrm{~mm}$ thick corrugated iron \\
\hline
\end{tabular}

\section{DESIGN SPECIFICATIONS}

The objective tasks and environment need to be defined before deriving the design specifications of the disaster-responding system. The test scenarios are defined after consulting experts and analyzing the environment for disaster situations. The chosen scenarios were a series of events that could occur while rescuing people from the site of a building collapse-a common situation in disasters.

A building site is an uneven terrain with a pile of building debris inclined in different directions. Therefore, the driving scenario involved accessing the site after rapidly overcoming various terrains such as irregular road surfaces, slopes, and stepped obstacles.

The work scenarios involved lifting large debris, crushing cement debris, and cutting steel bars to secure an access road to transport equipment or firefighters to the site. The scenarios also include lifesaving actions like safely removing debris accumulated on buried people, spreading out structures such as doors or walls to secure an exit for trapped people, and pushing debris.

The required tasks and motions were classified according to the aforementioned driving and work scenarios. The objective specifications for each mission are defined in Table 1.

The design specifications of the disaster-responding special-purpose machinery were derived through defined task objectives and existing equipment analyses, based on the following scenarios.

- Maximum reach: The distance from the center of the main frame's swing bearing to the attachment mounted in the manipulators must be equal to $6 \mathrm{~m}$.

- DOF: The one-arm manipulator joint, attachment, and leg joints must have 7 DOF, 1 DOF, and 2 DOF, respectively.

- Lifting force: The system must be capable of lifting 1 ton per arm and 400kg, including the attachment at its maximum reach.

- Driving speed: The maximum driving speed of the complete system on a flat land must be $4.1 \mathrm{~km} / \mathrm{h}$.

- Climbing ability: The maximum angle at which the complete system can climb while maintaining the position of the mainframe must be $30^{\circ}$

- Turning radius: The system must be able to turn within a radius of $1.65 \mathrm{~m}$ from its center. 


\section{SYSTEM DESIGN}

\subsection{Dual-Arm Hydraulic Manipulator}

The manipulator shape must be designed according to the controllability of the system as it uses a combination of the driver's movements and robotic control technology. In addition to performing various tasks in an unstructured environment that meets the aforementioned design specifications, the manipulator system must perform two tasks simultaneously or individually and perform the same task in different ways. These include cutting a steel bar with one arm while grabbing it with the other or lifting an object that is difficult to grab with one arm by using both arms. Owing to their aforementioned versatility, dual-arm systems are being researched extensively.

The dual-arm manipulators were designed to ensure that the two arms can work together while conforming to the design specifications and conditions. When joints are set up by using hydraulic actuators, the range of motion $(\mathrm{ROM})$ is relatively limited $90^{\circ}-110^{\circ}$ in general. This joint structure arrangement affects the performance of the manipulators. To overcome this problem, the joint arrangement design was optimized by analyzing the work area of the dual-arm manipulators through the product of the total and common workspace area technique (PTCWA) proposed by Bagheri in a study that presented the specifications of the hydraulic actuator and the joint construction method (Bagheri, Ajoudani, Lee, Caldwell, \& Tsagarakis, 2015). The joint structure of the manipulator in the current study was designed according to the results of the study carried out by the previous study (Cho et al., 2016), as shown in Fig. 2. $\left\{B_{s}\right\}$ is the reference coordinate of the dual-arm work manipulator. Joints $J_{1}-J_{4}$, designed for translation motion, had a YawPitch-Roll-Pitch structure, and joints $J_{5}--J_{7}$ had a Pitch-Yaw-Roll structure to replicate the human wrist joint and its RoM. The ROM, piston diameter, and the joint torques of the designated joints are listed in Table 2.

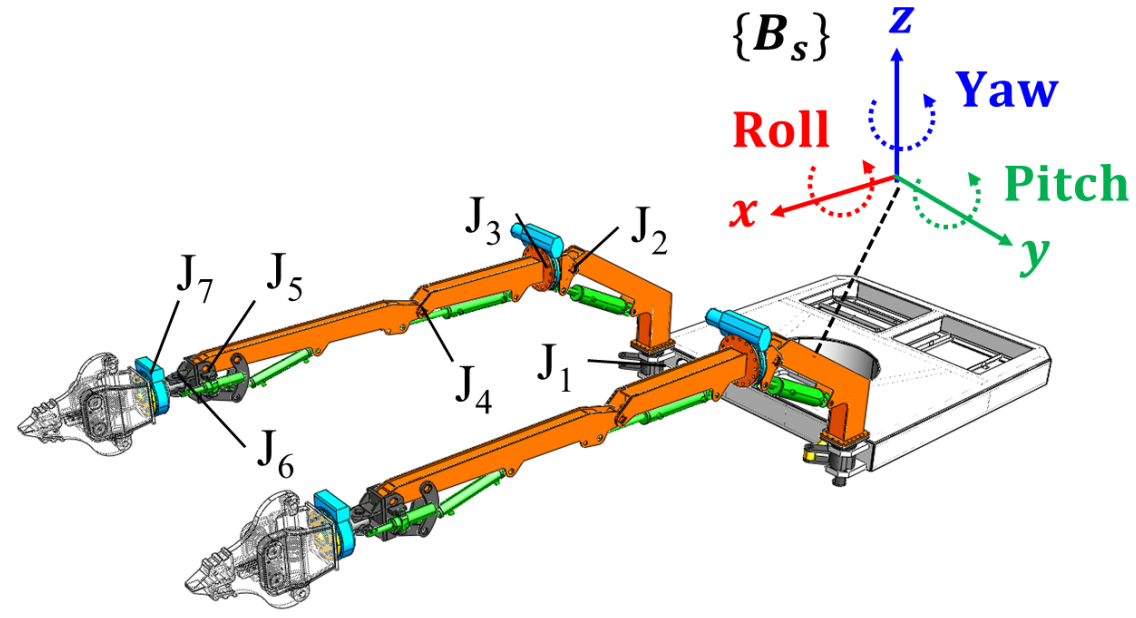

Figure 2: Design of Dual-Arm Hydraulic Manipulator

A double-acting piston, which is a commonly used hydraulic cylinder in excavators, is primarily used in the joints of the dual-arm manipulator. The joint mechanism needs to be designed according to the working angle of the joint, and the actuator must account for the direction of gravity due to the difference between the tensile and compressive force. 
Table 2: Specifications of Each Joint

\begin{tabular}{c|c|c|c}
\hline Joint & Range of Motioin $\left[^{\circ}\right]$ & Diameter of the piston $[\mathrm{mm}]$ & Torque $[\mathrm{kNm}]$ at 140 bar \\
\hline$J_{1}$ & $-27 \leq \theta_{1} \leq 63$ & 100.0 & 26.0 \\
$J_{2}$ & $-30 \leq \theta_{2} \leq 60$ & 90.0 & 22.7 \\
$J_{3}$ & $-70 \leq \theta_{3} \leq 70$ & - & 4.2 \\
$J_{4}$ & $0 \leq \theta_{4} \leq 100$ & 70.0 & 11.7 \\
$J_{5}$ & $-30 \leq \theta_{5} \leq 110$ & 50.0 & 10.1 \\
$J_{6}$ & $-45 \leq \theta_{6} \leq 45$ & 50.0 & 4.0 \\
$J_{7}$ & $-90 \leq \theta_{7} \leq 90$ & - & 1.0 \\
\hline
\end{tabular}

We confirmed the design specifications of the dual-arm manipulators in the previous study (Kim et al., 2020). The distance between the swing bearing center and the attachment connected to the manipulators is $6 \mathrm{~m}$. The link connected to each joint was produced as a box-type welded structure. A structural analysis was carried out to ensure that each arm was capable of lifting 1 ton and an additional $400 \mathrm{~kg}$ at the manipulator's maximum reach. The position of the valve manifold block, which is shown in Fig. 3, was selected according to the positions of the driving joints and pipe connections as follows. In addition, a hydraulic pipe was installed to minimize interference and pressure loss during the functioning of the multiple joints.

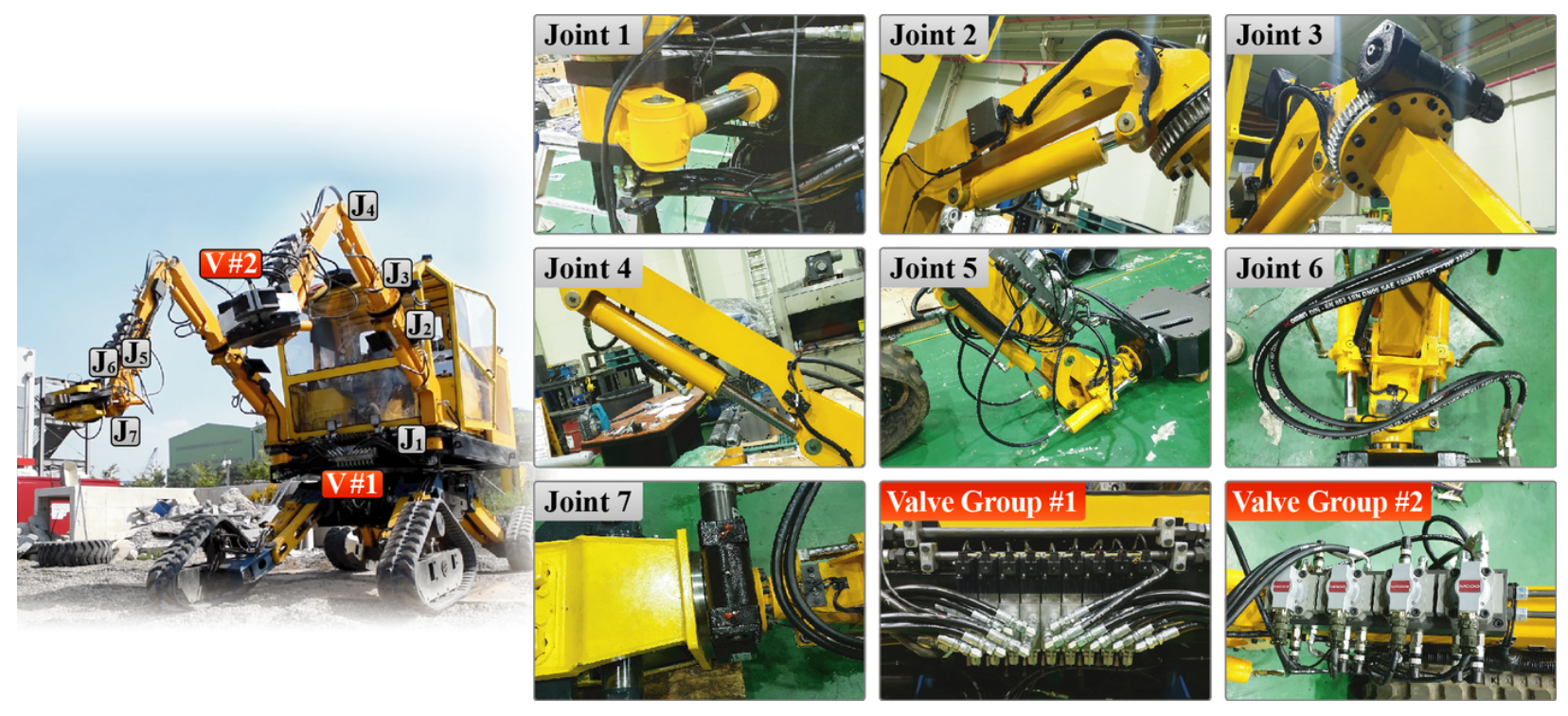

Figure 3: Configuration of the dual manipulator

The cylinder in $J_{1}$ is placed in the direction of the yaw axis according to the work performance of the two arms and the mechanical interference between them. The cylinder was mounted inside the mainframe to ensure that the end of the frame had a simple shape. This also created sufficient space to install 9-sections of Danfoss PVG valves, which are the control valves of the joints $J_{1}-J_{4}$ and the swing joint. The elements were designed to ensure the accurate movement of the entire manipulator while maintaining its stiffness. Joints $J_{2}, J_{4}$, and $J_{5}$ are placed in the direction of the pitch axis. These joints are placed along the direction of gravity. The actuators used for these joints are designed according to the work performance and RoM requirements. For instance, $J_{5}$ was designed to have a wide RoM of $140^{\circ}$ by using a joint mechanism similar to that of the 4-bar structure, and its actuators were designed for lifting tasks. Joints $J_{3}$ and $J_{7}$ 
rotate in the roll direction by using a hydraulic motor and a worm gear. They were modified to have a wide RoM in a compact design space. Joint $J_{6}$ rotates about the yaw axis by using two cylinders of the same size. Moog's G761 servo valve manifold is attached to the arm, which also serves as a control valve for joints $J_{5}-J_{7}$ and the attachment.

Every joint was mounted along with the non-contact sensor NRH300DP (Penny \& Giles, Davidson, North Carolina, USA) to measure the rotational angle. A specific mounting device was designed for this purpose while accounting for the hydraulic hoses and adapters that supply hydraulic fluid to every cylinder.

A multifunctional attachment for performing a variety of tasks was attached to the right manipulator, while a grab-type multi-branch attachment for grabbing atypical objects was mounted at the end of the left manipulator. The attachments were designed by using the TRIZ method to respond to different tasks in situations of disaster (Cho, 2018).

In a disaster situation, we don't know what kind of work will be done, we need to have multiple attachment devices to deal with various tasks. In the case of excavators, attachments without actuators, such as buckets, have a removable mechanism that allows drivers to exchange only with skilled control. However, in the case of attachments with actuators such as breakers, share, and crushers, hydraulic lines must be connected by humans even if it is connected by a mechanism. The removable type can be exchanged for various tasks, while the exchange takes a long time, and the removable mechanism causes it to be large and heavy. Therefore, we design the attachment to respond to various tasks by separating the necessary functions without replacing the attachment. In other words, the function that requires precise operation is on the right, the function of grabbing a variety of objects on the left, a device for holding a large object is placed in both arms. 


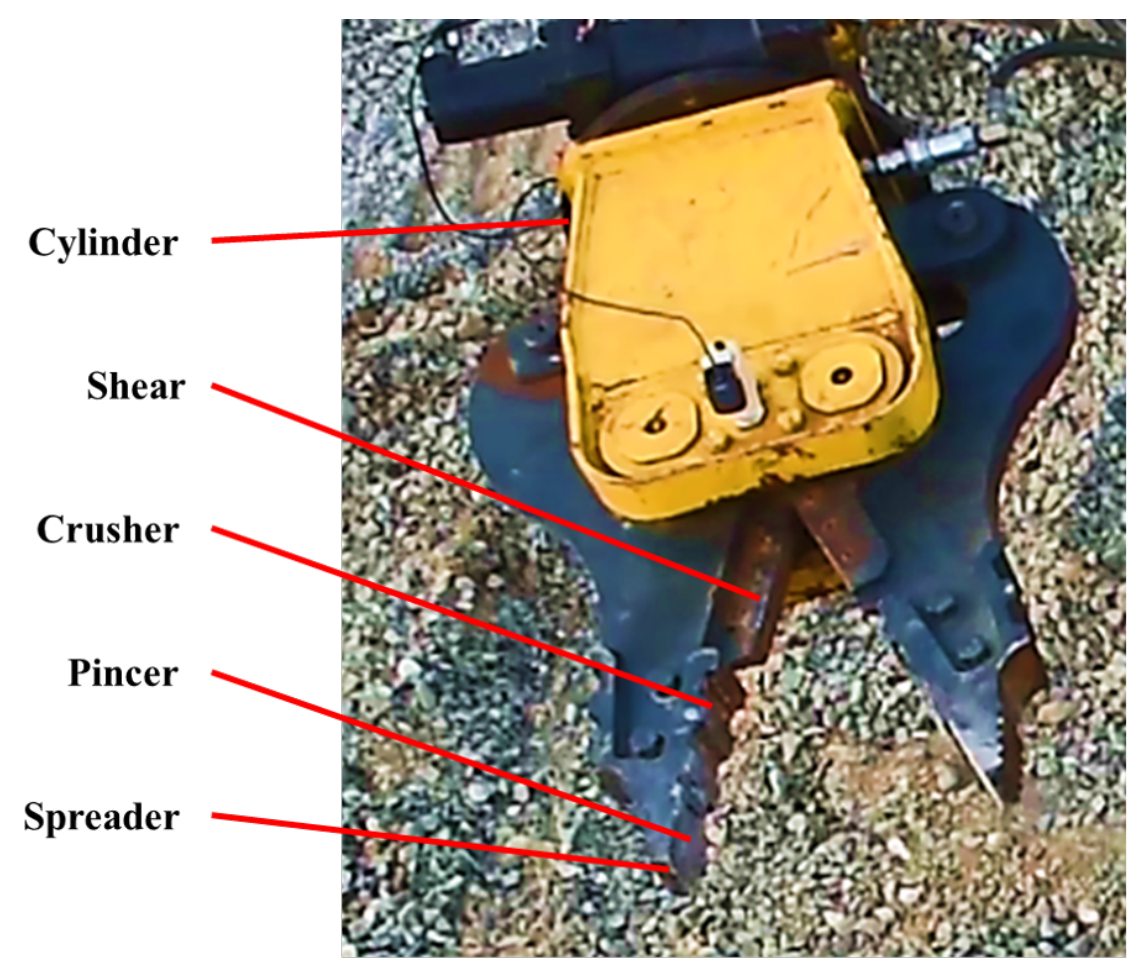

(a)

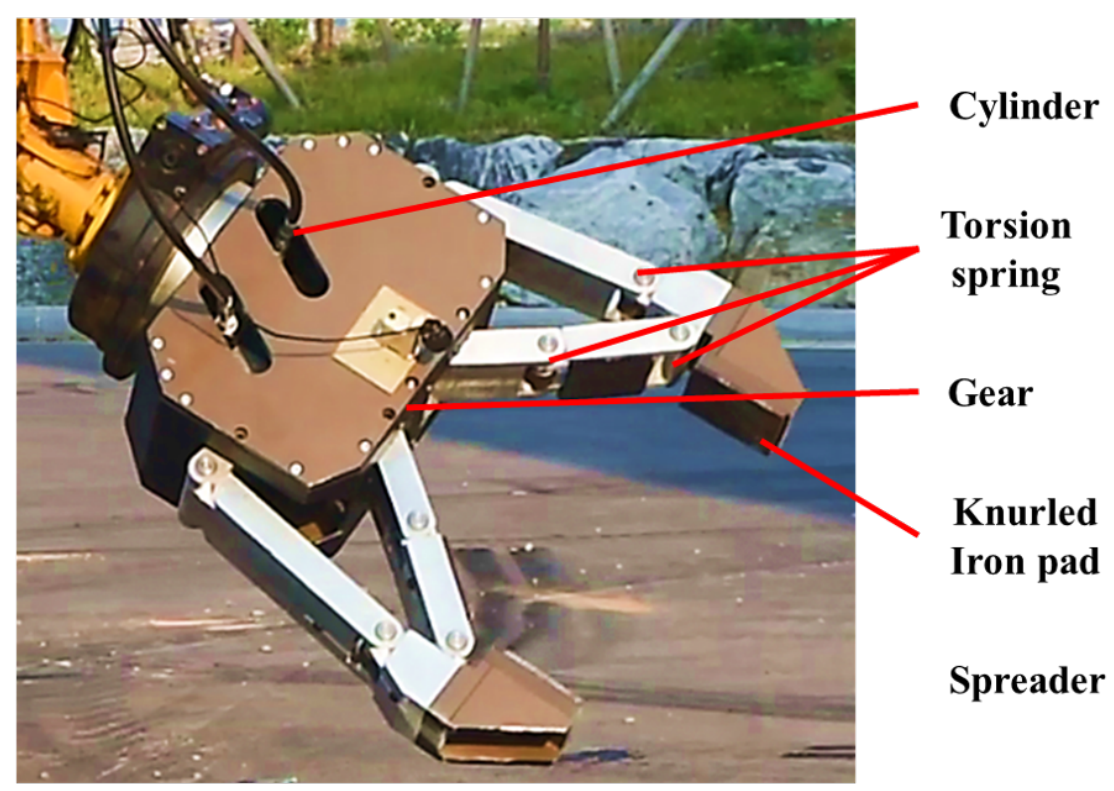

(b)

Figure 4: Configuration of attachments 
The right side is designed as a multifunctional attachment by tying together the tools needed for precise work. The mechanical structure of the multifunctional attachment is illustrated in Fig. 4(a). Not only need to cutting and crushing but also need for spreading the sandwich panel or open the debris. In addition, it was designed with the structure to increase the rotation function of steel bar or pipe with a diameter less than $40 \mathrm{~mm}$. The cylinder was selected based on a cutting force of $350 \mathrm{kgf}$ for cutting $\phi 20 \mathrm{~mm}$ rebar. Accordingly, it was designed to have a crushing force of about $220 \mathrm{kgf}$ and a grip force of $120 \mathrm{kgf}$, and the blade of the cutter was designed to be $150 \mathrm{~mm}$. It is necessary to adjust the gripping force in order to grip various objects of different materials with the pinching part. In this study, a method of controlling the cylinder pressure and controlling the required grip force was adopted. The multifunctional attachment has only 1 DOF and just two simple stages for gripper actuation, closing, and opening. In the current design, a linear actuator is used to actuate the attachment.

Grabs used in construction machinery have different gripping performances depending on the shape of the object. The reason for producing a dedicated grab is to improve the gripping performance and increase the efficiency of work. The finger type attachment was designed to allow gripping of objects of various shapes, even if only the pressure of the cylinder is controlled for unstructured objects. The linear actuator is located at the attachment base to actuate the finger-type attachment. The finger joints consist of a four-bar linkage and springs in a complex structure that can adapt to the shape of an object. As it can be seen in Fig.4(b) of the gripper, the finger is connected with a shaft and link mechanism to the actuation. It is possible to control the grasping force by controlling the pressure, and the spring allows for human-like grasping. The required specifications were designed with a gas tank $(367 \mathrm{~mm})$, C-shaped steel $(250 \mathrm{~mm})$, brick $(50 \mathrm{~mm} \times 200 \mathrm{~mm})$, and cement block $(250 \mathrm{~mm})$, with concrete debris of less than $400 \mathrm{~mm}$, and the weight of a graspable object was selected as $200 \mathrm{~kg}$.

\subsection{Driving System}

Disaster-responding systems must have a different mechanism from that of conventional excavators to overcome obstacles and quickly access the disaster site. In addition, a mechanism for maintaining the posture of the system must be present to enable roll, pitch, and yaw controls while keeping the workers safe and preventing the machinery from overturning while working on a slope. Therefore, to account for the roughness of the ground and the design requirements, the mechanism of the four legs was designed such that each leg could be moved up, down, left, and right through two cylinders (Shin, Ko, Seo, \& Hong, 2016)(Ha et al., 2016). The system can be raised by $1.2 \mathrm{~m}$ above the ground by controlling the first cylinder. The posture of the mainframe can also be controlled according to the ground condition. Since the horizontal distance of the crawlers can be widened by controlling the second cylinder of the leg, the system can be driven in a considerably stable posture while working or moving on unstable terrains without installing a separate outrigger, as shown in Fig.5(a). Consequently, the postures can be controlled up to an angle of $30^{\circ}$ along the roll axis and up to $25^{\circ}$ along the pitch axis. 


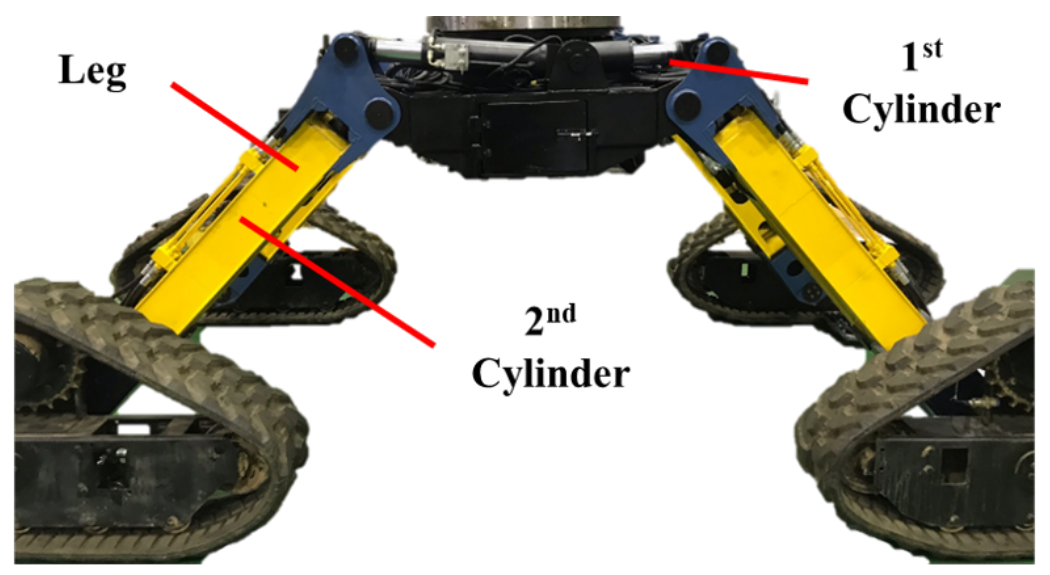

(a)

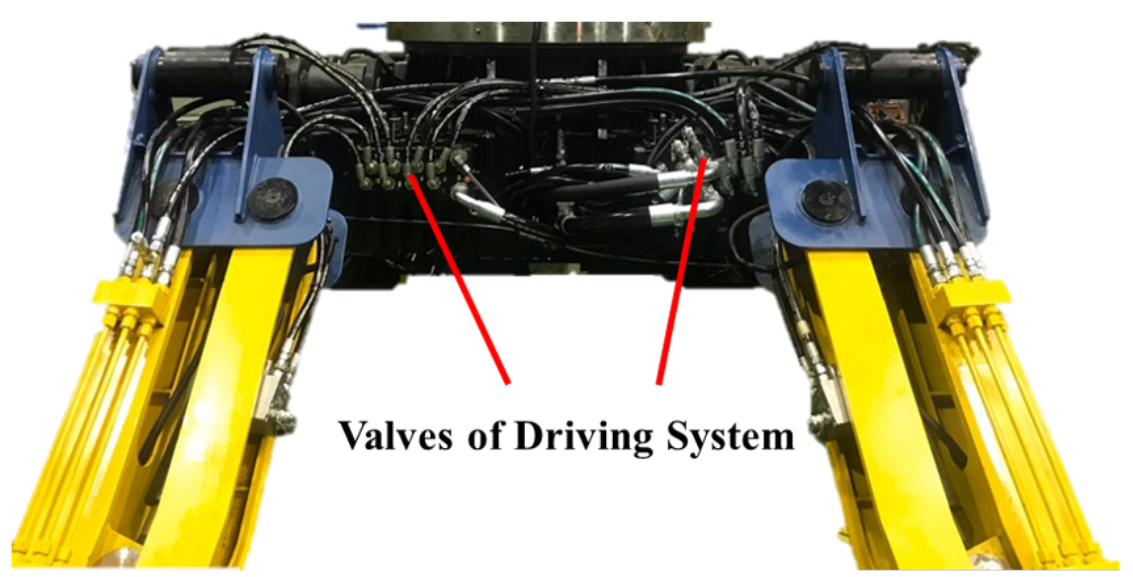

(b)

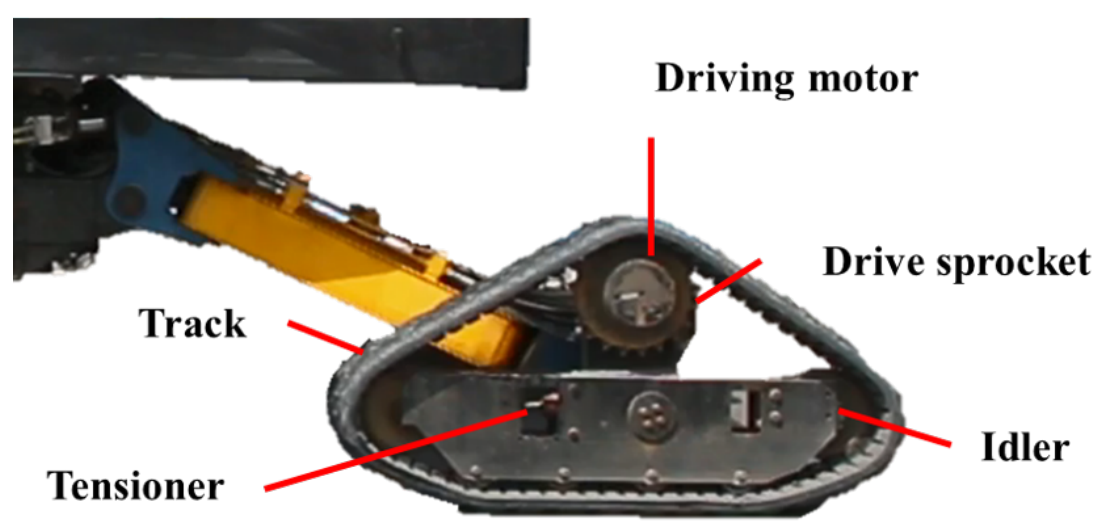

(c)

Figure 5: Configuration of the driving system 
The driving motor was selected to achieve a maximum driving speed of $4.1 \mathrm{~km} / \mathrm{h}$. The Danfoss PVG 32 valve was used to control the valves of $J_{1}$ and $J_{2}$ of each leg, and the Danfoss PVG 100 valve was used to control the motor driving the crawler. The control valves were placed on the front and back of the chassis of the driving system, as shown in Fig. 5(b).

The crawler structure consists of a track, driving motor, drive sprocket, idler, and tensioner, as shown in Fig. 5(c). The position of the pinpoint connected to the leg was defined through static analysis. The crawler mechanism was designed to provide posture stabilization. Torsion spring and stopper were designed at the connection point to prevent rotation of the crawler connected to the end of the leg.

\subsection{Cabin}

The special-purpose machinery is typically controlled by a person present in the cabin. Thus, it must be designed according to the driver's workspace, the field of view, and convenience. In addition, it is vital to consider the combination of the hydraulic line, which delivers hydraulic fluid from the power pack, with the mainframe to operate the dual-arm manipulators and the driving system.

The cabin was designed according to the work area of the controller used by the driver to control the entire system from its center. The outer frame of the machinery was designed with bent pipes and wire meshes. The size of the cabin, which was designed according to the workspace of the controller and the sizes of the mainframe and power pack, was equal to $1.78 \mathrm{~m} \times 1.44 \mathrm{~m} \times 1.5 \mathrm{~m}$, as shown in Fig. 6. The right side of the cabin was designed for placing the controller components, and the left side was designed to accommodate the uninterruptible power source (UPS) to supply power to the controller. The door was placed on the left side due to the positions of the dual-arm manipulators and the controller. The front portion of the cabin was designed according to the viewing angle of the driver while working with both arms. Polycarbonate was applied to the chassis to protect it from external materials and noise. At the bottom of the cabin, sufficient space was retained for the swing motor and the hydraulic hoses connected to the dual-arm manipulators and the driving system. In addition, a fastening component was designed according to the cabin mounting method.

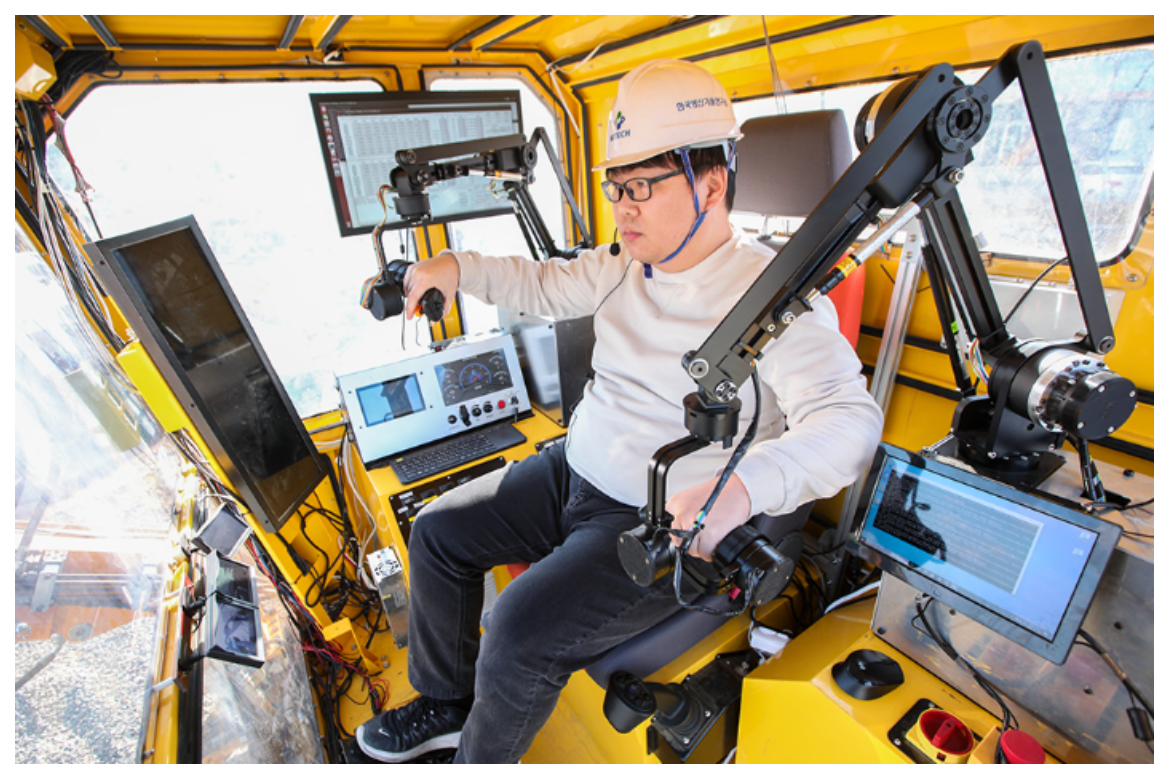

Figure 6: Configuration of Cabin 


\subsection{Hydraulic System}

The specifications of the power pack were defined according to the requirements of a machine weighing 5 tons. The engine and pump models were selected according to the results of the hydraulic simulation, the performance of functioning machinery, and future commercialization requirements (Lee, Noh, Jang, \& others, 2018)(Choi, Le, \& Yang, 2019). The Doosan D24 engine was chosen for the proposed machinery. It is a 4-cylinder turbo-diesel engine with a power output of $55 \mathrm{~kW}(75 \mathrm{HP})$ and satisfies the Tier 4 Final emission regulations. This ensures that the system would be commercially viable in the future. Two remote pressure-compensated axial piston pumps, which are part of Danfoss' Series 45 pumps, were connected to the system. Pump 1 was used to drive the dual-arm manipulators and the drive motor, while Pump 2 was used to drive the legs of the driving system. Pump 1 has a maximum pressure and flow rate of 230 bar and $71 \mathrm{~cm} 3 / \mathrm{rev}$, respectively, and Pump 2 has a maximum pressure and flow rate of $250 \mathrm{bar}$ and $25 \mathrm{~cm} 3 / \mathrm{rev}$, respectively. The power pack includes the engine, engine control panel, battery, cooling system, hydraulic pumps 1 and 2, housing and coupling for establishing connections, and hydraulic parts for generating and supplying hydraulic pressure, as shown in Fig. 7. It also has brackets to fix the fuel tank, hydraulic oil tank, and hydraulic block valve. The volume of the hydraulic oil tank was chosen to be 140L on the basis of the predicted flow rates of the special-purpose machinery. In addition, a radiator and a cooling fan were installed to cool the hydraulic oil.

Unlike construction machinery, which focuses on work efficiency, disaster equipment requires its hydraulic system control to vary the force required, depending on the nature of the work. The pump pressure is set according to the force required for the task, and the engine's rated speed is set according to the maximum work speed of the task. The dual-arm manipulator uses a proportional electronic pressure compensation control method that allows the rider to set the pump pressure and maintain it according to the characteristics of the task and its corresponding force requirements. In addition, a flow control method linked to the engine load was adopted to minimize the engine stops and engine speed fluctuations due to variations in the instantaneous workload.

The load sensing method was adopted to control the leg posture and the driving motor of the driving system. Load sensing control is a method of supplying the minimum required flow rate and pressure to the actuators and the motor while mechanically controlling the pump discharge to achieve a constant pressure difference across the flow control valve next to the pump. Thus, these designs allow special purpose machinery to appropriately distribute the hydraulic pressure according to the load while working and moving on uneven terrain. This allows the machinery to deliver an optimal performance at disaster sites. 


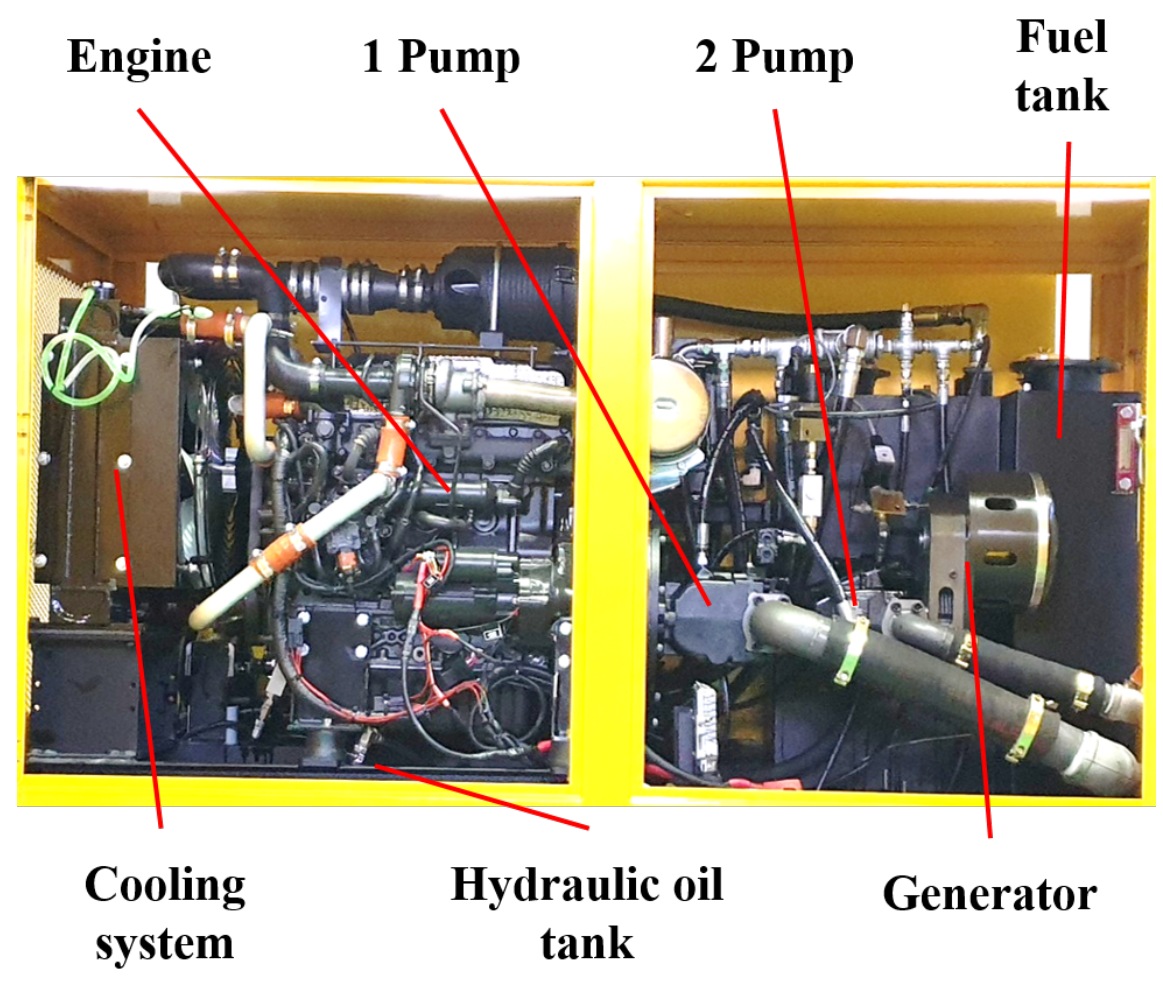

Figure 7: Configuration of the power pack

\subsection{Control Network System}

The actuators and sensors in the dual-arm manipulators were grouped by element as follows, (L/R) wrist, $(\mathrm{L} / \mathrm{R})$ arm, and center. A distributed interface was used to exercise real-time control over the dual-arm manipulators. The series of actuators and sensors were then optimized to function as an efficient combination of a CPU and an embedded system, as shown in Fig. 8. This allowed the computational processes and the commands to be performed rapidly and intensively at the center. In addition, a control network system was developed by using EtherCAT (100 Mbps), UDP (100 Mbps), and RS485 (10.5 Mbps) communications.

The CPU used for the GUI and control operations of the system is an Intel Haswell i5-3.4 GHz processor, with $16 \mathrm{~GB}$ of RAM and a $128 \mathrm{~GB}$ SSD and acts as the EtherCAT master. It controls the EtherCAT communication system and facilitates socket communication between the dual-arm manipulator control devices in the cabin. It is an RTOS system that has patched Xenoma to Ubuntu Linux and uses wxWidgets as its GUI. This system sends and receives data required to control the dual-arm manipulators by using socket communication and manages all the controls at the center, including algorithm operations, sensor data processing, and the commands required to control the system by using the EtherCAT communication.

EtherCAT communication was used for the high-speed real-time transfer of a large number of packets from the upper/lower bodies to the center. This system also uses RS485 communication to transfer a small number of packets across a distributed interface of devices by element. It is lighter than EtherCAT and can still carry out multiple high-speed communications. The upper body embedded board, which uses the STM32F407 MCU, and the ECS-E100 lower body embedded board act as the EtherCAT slave and communicate with the EtherCAT master PC. The bridge embedded board, which uses the STM23F405 
MCU, plays the role of the master of the RS485 communication system and communicates with local joint control units (JCU). In addition, a dual-port RAM (DPRAM) was used to exchange collected data by using heterogeneous communications between the EtherCAT slave and the bridge board.

The JCU-embedded board, which uses the STM32F405 MCU, collects information from multiple sensors located throughout the robot in five parts by element and drives the hydraulic actuators through the control inputs transmitted from the PC. In this board, five JCUs are connected through the RS485 communication system.

The developed control network system was optimized for the dual-arm manipulators at a relatively low cost. It could perform real-time high-speed communications in control cycles of $1-2 \mathrm{kHz}$. It was also capable of generating quick and flexible responses to user requests through the universal RS485 communication system and the distributed interface design by element.

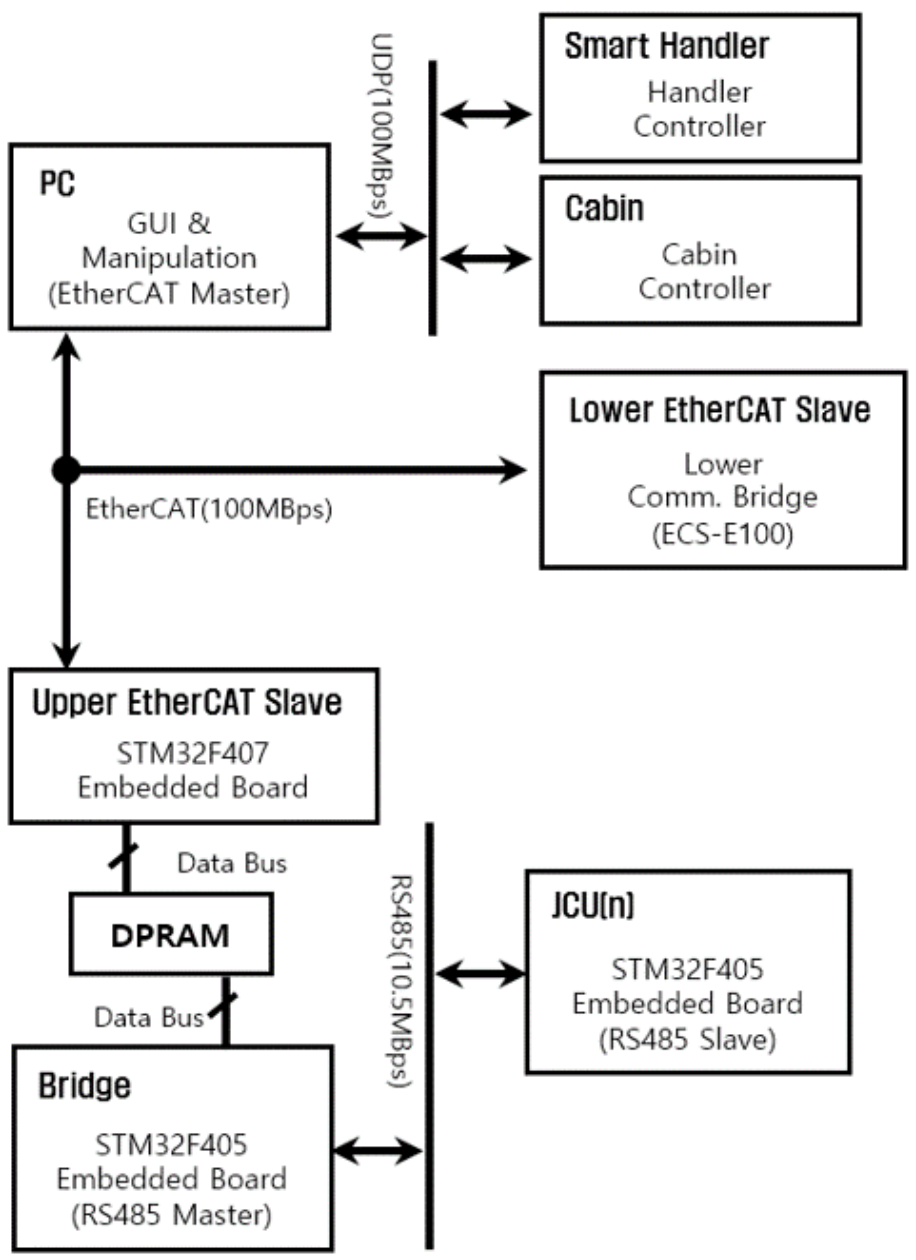

Figure 8: Structure of the Control Network System 


\subsection{System Integration Design}

System integration refers to the integration of a series of processes, including the development, construction, and operation of systems. We designed the overall layout of the system to optimize the operations according to the design objective of each component. The system was further optimized for weight distribution to prevent it from overturning. This was carried out by accounting for the total weight, testing the functions of each component, and conducting simulations to ensure the workability of the overall system in different scenarios. Through this process, the binding positions of the mainframe, dual-arm manipulators, driving system, cabin, and power pack were determined.

The mainframe, which is where all the modules are combined, was designed by performing a stiffness analysis simulation to obtain the stiffness that would be sufficient to carry out the specified operations. It was fabricated as a welded structure. The mainframe was connected to the driving system through a swing bearing and a center joint to enable free rotation. The center joint was designed to supply the hydraulic pressure required for controlling the leg and rotating the crawler of the driving system. This hydraulic pressure was generated by the power pack. The swing bearing was connected between the mainframe and the driving system. It had sufficient durability in dynamic conditions and also accounted for the maximum load condition of the manipulators and the weight of the system. The design of the cabin mount device accounted for the interference caused between the swing motor, power pack, and cabin mounting method. The components were arranged according to the center of gravity and the overall height of the system. The rotation of the hydraulic hose, power line, and communication line were prevented by mounting the center joint and slip ring.

The engine power pack system was installed in the mainframe. It consisted of hydraulic components to drive the machinery. The spaces in the mainframe were designed to place and mount components like the engine, pump, cooling device, hydraulic oil tank, and fuel tank. The system exterior comprises a cover chassis to protect various components from external shock.

\section{CONTROL ARCHITECTURE}

The special-purpose machinery proposed in the current study comprises 14 DOF dual-arm manipulators, swing joints, attachments, four legs, and crawlers. This machinery requires a system to provide the driver with sufficient control to perform complicated tasks by manipulating the machinery. Different manipulation and control methods adopted by existing excavators that move one joint with one lever are needed to control the dual-arm manipulators simultaneously. The control system used to control the movements of the left and right manipulators with the left and right controllers was designed by combining the driver's movements with robotic control technology. In robotics, human control of the robot arm is referred to as the master-slave control. The control algorithm from our previous study was configured to enable the dual-arm manipulators to perform tasks by moving according to the movement of the two arms of the driver holding the master device, as shown in Fig. 9. The Marionette algorithm for intuitive control of 'as if the operator's arm is moving' was presented as a control strategy for dual-arm manipulators with attachment (Kim, Park, Han, Kim, \& Jo, 2020). 


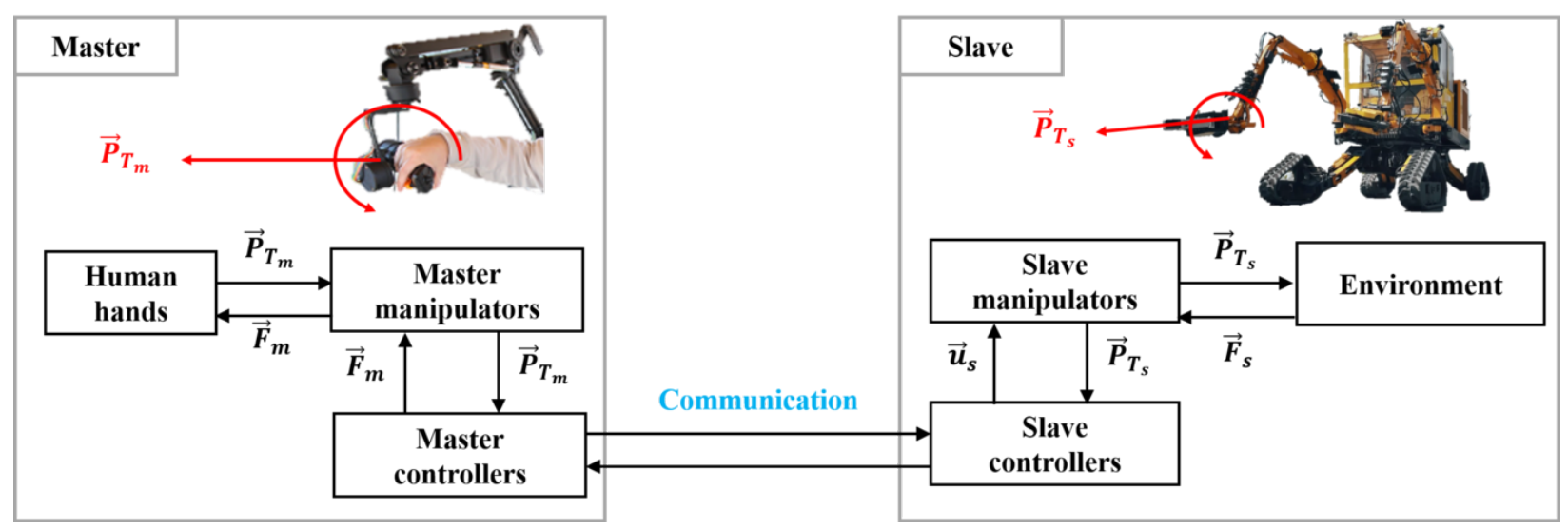

Figure 9: Block diagram of Control Strategy

We developed a master device. The joystick can be held by a human hand and pushed in the desired direction on the reference coordinate $\{m\}$ to generate an input along the $\mathrm{x}, \mathrm{y}$, and $\mathrm{z}$ axes. The ROM of this controller was designed according to the range of convenient movement of both arms of the driver in the cabin. Since the wrist of the controller has 3 DOFs, it is possible to generate inputs in the roll, pitch, and yaw directions on the reference coordinate $\{m\}$. Thus, the operator can generate inputs in certain directions by moving the controller to the desired position.

The driving system comprises four 2 DOF legs, with an independently driven crawler attached to the end of each leg. An initial rapid response cannot be carried out by controlling each leg or using the manipulators to move on an uneven surface in a disaster situation. A simpler method for moving while performing tasks is required because both arms of the operator are holding the controllers of the manipulators. Therefore, we have added four pedals to control the driving system. The left/right pedal A can move back and forth from its initial position. The left and right pedals A were allocated to generate forward and reverse inputs for the two left and two right crawlers, respectively. This allows the implementation of forward/reverse and turn operations. In addition, provisions are made to adjust the height of the machinery. The right and left pedals $\mathrm{B}$ were allocated to generate an upward and downward input, respectively. The system was designed to control the height by simultaneously moving the legs and the crawlers. In addition, the system was designed to move while maintaining the posture of the mainframe on a slope without separate leg control through a hydraulic system composition that includes a load sensing pump and an algorithm to control the leg joints according to the load of each leg.

\section{EXPERIMENTS}

This chapter describes the tests performed at the test site to evaluate the performance of the special purpose machinery in disaster scenarios. In order to create lifesaving work scenarios and representative work modes at building collapse sites and use them to evaluate system performance, we developed a test site, based on a simulated disaster environment, for performance evaluation, as shown in Fig. 10. We have simulated events that may occur in lifesaving rescue tasks at disaster sites as a series of processes and defined tasks by using the work modes according to the target specifications of the special purpose machinery. In a situation wherein a person is trapped under collapse debris and needs to be rescued from a building collapse site, the machinery overcomes the rough environment and reaches the target point through the driving scenario. The 
buried person is then rescued by using the machinery to safely removing the collapse debris through the work scenario. The work target specifications of each mode are outlined in Table 1.

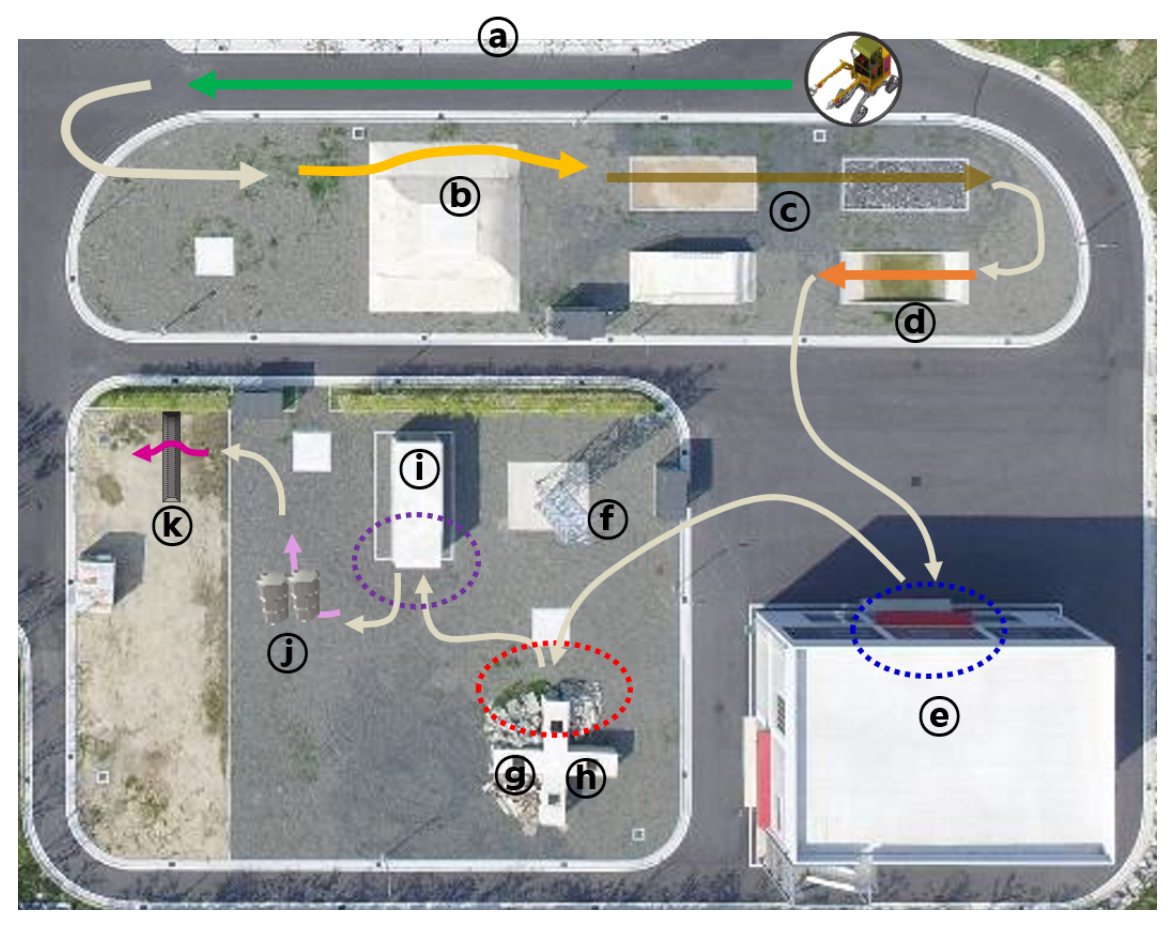

Figure 10: Scenario test site: (a).Flat surface, (b).Lateral slope, (c).Uneven surface, (d).Longitudinal slope, (e).Lifting pipe, (f).Lifting tire, (g).Crushing brick, (h).Cutting rebar, (i).Tearing of sandwich panels, (j).Pushing drum barrel, (k).Overcoming obstacle

The results of flat ground driving, among driving scenarios, are shown in Fig. 11(a). The operator drove on the machinery on a $60 \mathrm{~m}$ flat ground made of asphalt by using the forward/reverse pedal A in the cabin. The speed was measured by using a prism that was mounted on the cabin. The prism reflects a laser beam, which measures the change in position. The driving speed was found to be $4.1 \mathrm{~km} / \mathrm{h}$.

Figure 11(b) shows the result of driving on a slope. The machinery maintained the horizontal orientation of the mainframe while driving with the left and right crawlers at low and high points on a $15^{\circ}$ lateral slope. In addition, the mainframe was kept horizontal when the crawlers entered the $11^{\circ}$ vertical slope, as shown in Fig. 11(c). At this time, the operator only controlled forward/reverse pedal A. This result reveals that the algorithm for adjusting the leg joints according to the loads on the hydraulic system, including the load-sensing pump and every other pump, is working.

For rough ground driving, a task of driving through a ground consisting of debris, which is $10 \mathrm{~cm}$ or smaller, was performed, as shown in Fig. 11(d). At this time, the operator only controlled forward/reverse pedal A, and the stable driving characteristic of the machinery was verified.

The difficulty of the task was increased by including steel frame obstacles with a height of $50 \mathrm{~cm}$ and a slope angle of $37^{\circ}$, as shown in Fig. 11(f). The machinery also passed over the installed obstacles safely. 

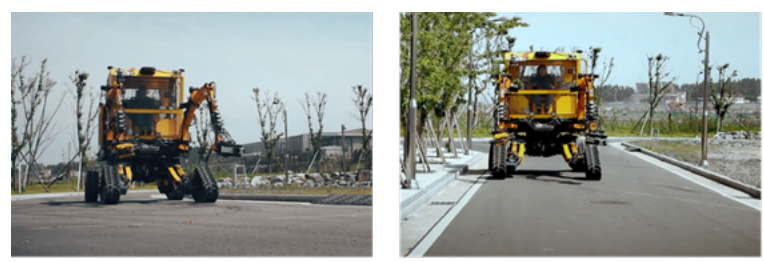

(a)
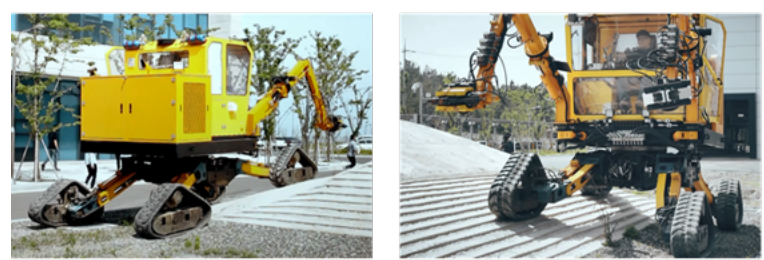

(b)
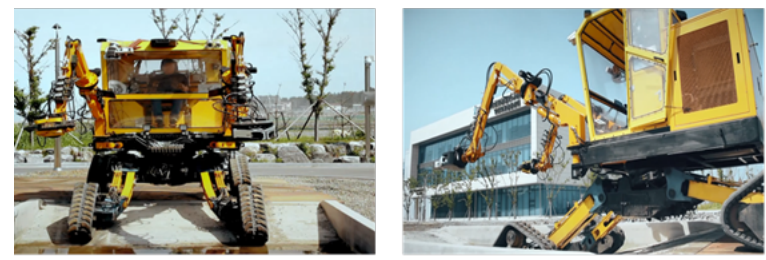

(c)
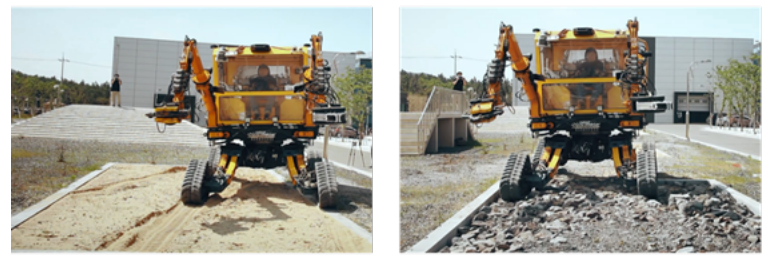

(d)
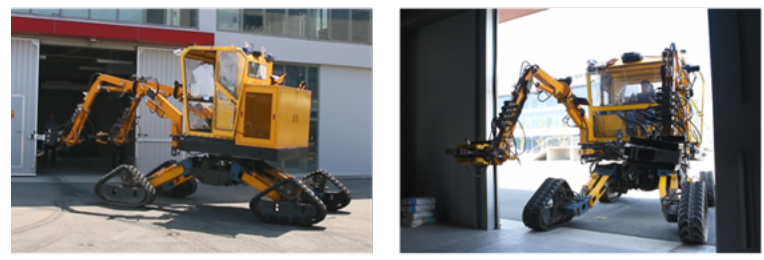

(e)
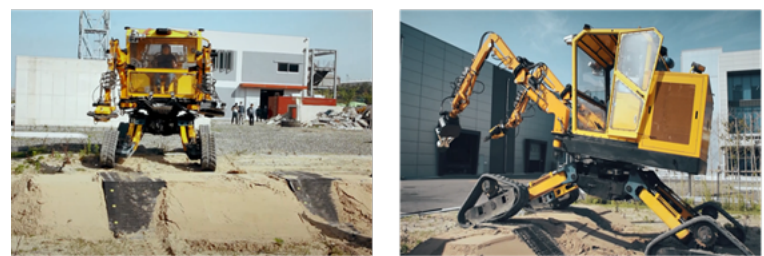

(f)
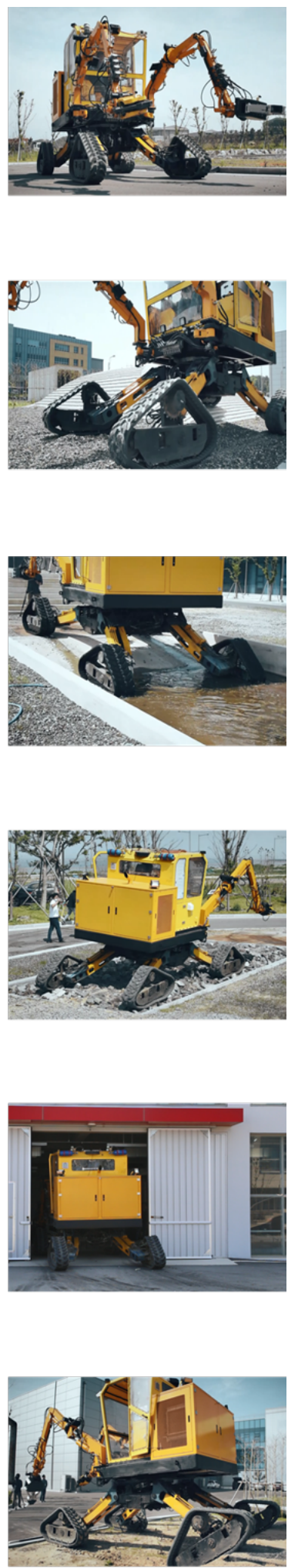

Figure 11: Driving test results 
A test was performed for a situation where a person is lying under a pipe in a factory and the special-purpose machinery enters the factory and supports the rescue task, as shown in Fig. 11(e). First, to pass through the factory gate and enter the factory, the height of the machinery was lowered by controlling pedal B. Then, the operator approached the buried person by controlling pedal A and removed the pipe from the buried person, as shown in Fig. 12(a).

A snapshot of the lifting process is shown in Fig. 12(b). A task of picking up a 200kg tire on the driving path, rotating and moving it with a swing joint was performed. In addition, a test was conducted to verify whether the design specification for lifting 1 per arm and lifting $200 \mathrm{~kg}$. The position and orientation of the attachment were adjusted to grasp the inner surface of the tire. After lifting the tire, the swing joint was moved to remove the tire in the driving path. In the case of objects that are difficult to grasp with one arm, both arms can be moved to work as shown in Fig. 12(c). It is a task to operate both arms simultaneously to position both ends of the drum barrel and lift both arms by applying force in the direction toward the object. This test verified the lifting power of the fabricated dual-arm manipulators, and it can be observed that the developed operating method allows the operator to manipulate the swing joint while performing a task by controlling dual-arm manipulators through the smart controller.

The result of performing the crushing work mode is shown in Fig. 12(d). The manipulator was operated to position the brick between the crushers of the multifunctional attachment, and then the brick was crushed by the attachment. The results of the cutting task are shown in Fig. 12(e). The operator controlled the left manipulator to grasp the rebar and simultaneously controlled both arms to position the rebar at the location of the shear of the multifunctional attachment of the right manipulator. And it was confirmed that the rebar was cut by manipulating the multifunctional attachment. It can be observed that the seven joints move simultaneously to move the endpoint of the manipulator to the target point, instead of moving each joint separately by the operator. This confirms that the endpoint position of the 7 DOF manipulator can be controlled intuitively using the proposed controller of the dual-arm manipulators and that concrete blocks can be crushed using the attachment. It also confirmed that it can grasp and move an object and simultaneously perform other tasks such as cutting another manipulator through the operating method.

The results of the spreading task are shown in Fig. 12(f). It can be observed that space is secured by drilling and breaking through a wall blocked by $0.5 \mathrm{~mm}$ thick corrugated iron using the multifunctional attachment to secure access and rescue passage for firefighters.

The results of the pushing task are shown in Fig. 12(g). This task involves pushing a drum on the ground to a distance by simultaneously controlling the swing joint and dual-arm manipulator. This shows that the endpoint of the manipulators and the swing joint can be easily controlled simultaneously. 

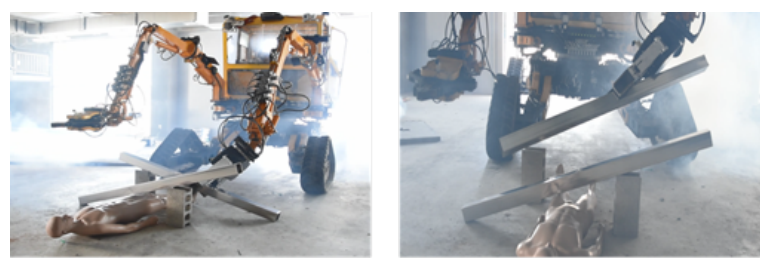

(a)
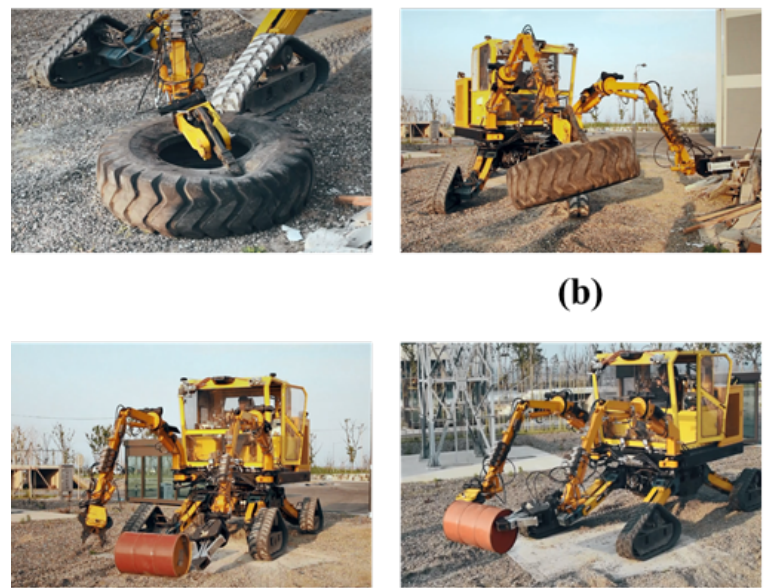

(b)

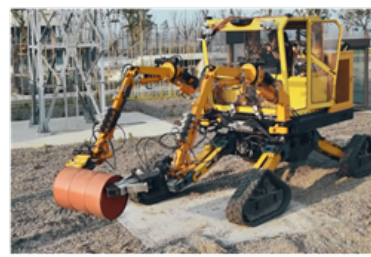

(c)
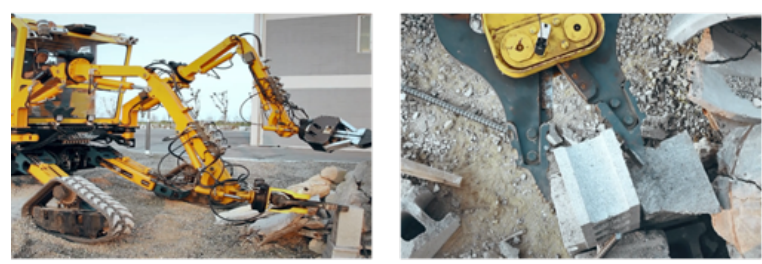

(d)
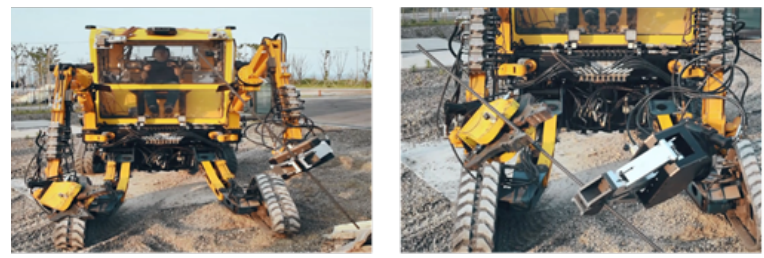

(e)
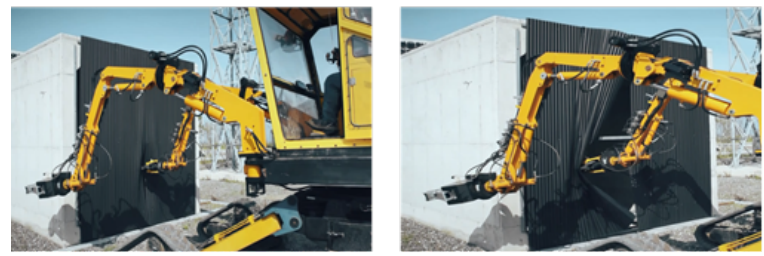

(f)
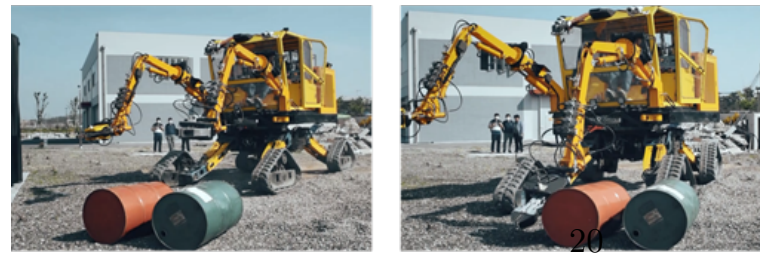

(g)
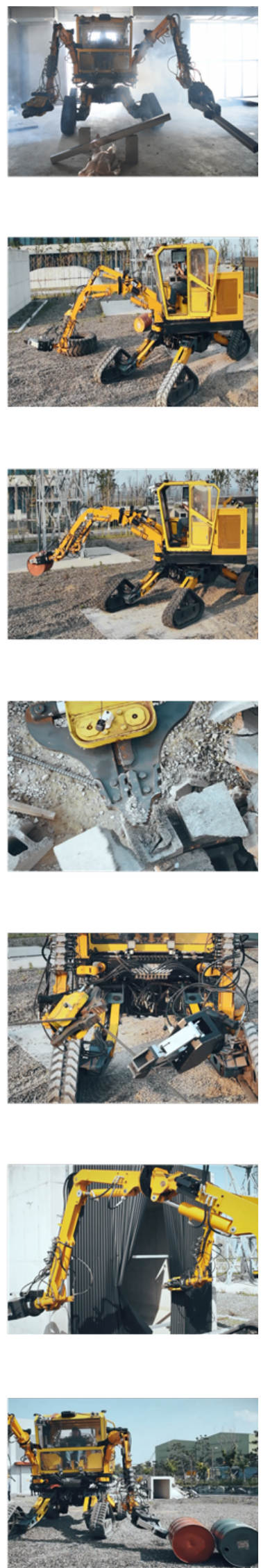

Figure 12: Working test result 
The above test results verified that one operator can ride and control the special-purpose machinery. Furthermore, the performance of the developed special-purpose machinery was evaluated by performing predefined tasks in a disaster environment.

\section{CONCLUSION}

This study describes the design and fabrication of special-purpose machinery for lifesaving and recovery support for a rapid initial response within the golden hour after the occurrence of disasters. We derived the design parameters by defining tasks through the analysis of scenarios in disaster-responding situations and existing special-purpose machinery. Based on the results of this analysis, the special-purpose machinery was fabricated, and its components were described in detail, including dual-arm manipulators, leg-crawlers, mainframe, cabin, power pack, and control networks. In addition, we introduced a system for operators to intuitively operate a system comprising dual-arm manipulators and four legs with crawlers attached to the end of the legs.

The tests performed at a test site for evaluating performance based on disaster scenarios conducted to verify the work performance of the integrated special-purpose machinery were described. The tasks were largely classified into the driving mode and work mode. The results confirmed that the rough environment could be overcome and the target point reached through the driving mode, and the collapse debris was safely removed through the work mode. These tests verified that one operator can ride and control the developed specialpurpose machinery, and the performance of the developed special-purpose machinery was demonstrated through the defined tasks.

\section{Acknowledgment}

This work was supported by the Technology Innovation Program(10052968, Development of core components and systems for special purposed machine in disaster environment) funded By the Ministry of Trade, Industry \& Energy(MOTIE, Korea) and in part by the Korea Institute of Industrial Technology as "Development of industrial robot and post-processing robot application technology for Ppuri industry (KITECH JH210015)" project.

\section{References}

Retrieved from https://www.hitachicm.com/global/businesses/products/double-arm-workingmachine/

Retrieved from https://www . youtube.com/watch?v=we18ahVF07E

Retrieved from https://www . youtube.com/watch?v=YD4w2e69vUk

Bagheri, M., Ajoudani, A., Lee, J., Caldwell, D. G., \& Tsagarakis, N. G. (2015). Kinematic analysis and design considerations for optimal base frame arrangement of humanoid shoulders. 2015 IEEE International Conference on Robotics and Automation (ICRA), 2710-2715. IEEE.

Cho, J.-S., Park, S.-S., Kim, J.-T., Park, B.-Y., Bae, J.-H., Lee, D.-H., . . Park, S.-D. (2016). Design of Dual Arm Manipulator for a Disaster-Responding Special Purpose Machinery. Journal of Drive and Control, 13(4), 95-101. 
Cho, J. S. (2018). Design of Attachments for Dual Arm of Disaster-Responding Special Function Machinery by TRIZ. Journal of Drive and Control, 15(3), 29-35.

Choi, S. W., Le, Q. H., \& Yang, S. Y. (2019). A Study on Hydraulic Simulation Analysis of a 7 DOF Dual Arm Machinery. 2019 23rd International Conference on Mechatronics Technology (ICMT), 1-4. IEEE.

Ha, J. W., Kim, S. H., Lee, Y. S., Sun, D. I., Jang, S. H., \& Han, C. S. (2016). A Study on the Modeling of Independently Driven Quadruped Crawler System for Posture Control of Special Purpose Machine for Disaster Response. Journal of Drive and Control, 13(4), 82-88.

Ishii, A. (2006). Operation system of a double-front work machine for simultaneous operation. Proc. Int. Symp. Automation and Robotics in Construction, 539-542.

Jeong, D., Kim, C., Kim, J. H., Suh, J., \& Jin, M. (2017). Mission Scenario-based Design of Hydraulic Manipulators for Armored Robot Systems. Journal of Drive and Control, 14 (4), 51-60.

Kim, J.-T., Park, S.-S., Han, S.-C., Kim, J.-H., \& Jo, J.-S. (2020). Control Strategy and Verification of Dual-Arm Manipulator for Disaster-Responding Special Purpose Machinery. Journal of Drive and Control, 17(4), 31-37.

Kim, J. T., Chon, S., Park, S., Kim, J., Lee, S., \& Cho, J. (2020). Experimental Investigation of a DisasterResponding Special Purpose Machinery. Conf. of The Korea Fluid Power Systems Society, 107-108.

Lee, G. H., Noh, D. K., Jang, J. S., \& others. (2018). Design feasibility study by analytical approach for a disaster response hydraulic driving system. Journal of Drive and Control, 15(2), 22-31.

Montazeri, A., \& Ekotuyo, J. (2016). Development of dynamic model of a 7DOF hydraulically actuated teleoperated robot for decommissioning applications. 2016 American Control Conference (ACC), 1209-1214. IEEE.

Nishida, T., Koga, H., Fuchikawa, Y., Kitayama, Y., Kurogi, S., \& Arimura, Y. (2006). Development of pilot assistance system with stereo vision for robot manipulation. 2006 SICE-ICASE International Joint Conference, 2675-2680. IEEE.

Park, S. H., Jin, M., Kim, Y.-R., Kim, D., Kim, J.-S., Shin, D. B., \& Suh, J. (2018). Vehicle body design of armored robot for complex disaster. The Journal of Korea Robotics Society, 13(4), 248-255.

Shin, D. B., Ko, Y. H., Seo, W. S., \& Hong, D. H. (2016). Design of a robot leg mechanism for early rescue and recovery support from a disaster. Journal of Drive and Control, 13(4), 89-94. 